\title{
Research status of different improvement measures for saline- alkali land
}

\author{
$\operatorname{Jian} \operatorname{Li}^{1,2,3, *}$ \\ ${ }^{1}$ Key Laboratory of Degraded and Unused Land Consolidation Engineering, Ministry of Natural Resources, xi'an 710021, China. \\ ${ }^{2}$ Shaanxi Provincial Land Engineering Construction Group Co., Ltd., xi’an 710075, China. \\ ${ }^{3}$ Institute of Land Engineering and Technology, Shaanxi Provincial Land Engineering Construction Group Co., Ltd., xi’an 710021, \\ China.
}

\begin{abstract}
Cultivated land is the fundamental guarantee of food security, and saline-alkali land affects the quality of cultivated land to varying degrees. It is of great significance to study saline-alkali land improvement measures to solve the problem of saline-alkali land utilization, promote the healthy development of agriculture and animal husbandry, and improve the quality of cultivated land. The author has sorted out the relevant literature and reports on the improvement methods and technologies of saline-alkali land at home and abroad in recent years, and introduced the status and effects of different improvement measures for saline-alkali land from four types: chemical improvement measures, physical improvement measures, water conservancy improvement technology and biological improvement measures. It provides a theoretical basis for the study of governance and saline-alkali land improvement measures.
\end{abstract}

Key words: Saline-alkali land; improvement measures; research status.

\section{Introduction}

Saline-alkali land is a general term for saline soil and alkaline soil. Its low organic matter content and poor soil fertility will seriously affect the survival and growth of plants, which has become a worldwide problem. In China, with population growth and the advancement of urbanization, arable land has become a precious and limited resource, and the existence of saline-alkali land has led to a decline in the quality of a large amount of arable land. At present, more than $20 \%$ of China's arable land is exposed to varying degrees of salinity. The influence of alkalization, with its large area and wide distribution, further stimulates the problem of contradiction between man and land. Therefore, it is an urgent problem to take effective measures to improve the salinized soil and make it can be effectively used.

In order to develop and utilize saline-alkali land, it is first necessary to find an effective method for controlling saline-alkali land. For a long time, how to reduce the degree of soil salinization, improve soil fertility, and use effective improvement measures to create a suitable soil ecological environment has been the focus of many experts and scholars [1]. The improved measures and mechanism research have achieved remarkable results, and some measures have achieved good results in practical applications and have been promoted [2]. Among them, the method of comprehensive management is the most effective, mainly as follows: prevention first and equal emphasis on prevention and control; comprehensive management and water conservancy first; unified planning and measures according to local conditions; In recent years, in addition to using traditional methods to transform soil, many experts and scholars have also begun to extend and transfer to the direction of cultivating salt-tolerant plants [3], and develop new crops and new technologies to improve the management of saline-alkali soils [4]. At present, the traditional improvement measures can be roughly divided into four major aspects: physics, chemistry, water conservancy and biology.

\section{Chemical Improvement Measures}

Chemical improvement measures have a wide range of applications, mainly using chemical reagents in salinealkali land to promote salt leaching, thereby improving soil structure. The main types of soil conditioners for saline-alkali land improvement include peat, organic fertilizer, perlite, vermiculite, humic acid, compound fertilizer, water retention agent, soil thickener, trace elements, bacteria, etc. The main types of saline-alkali land improvement solutions are Malay Acid anhydride, wood acetic acid, amino acid, humic acid, black diamond, trace elements, etc. According to the research results, the application of phosphogypsum can effectively improve the soil structure, and the application of polymerized

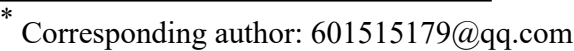


aluminum ferric sulfate, phosphogypsum, and desulfurized gypsum in saline-alkali soil can reduce soil $\mathrm{pH}$ and total alkalinity, eliminate soda salt, accelerate the desalination rate of saline-alkali soil, and reduce the generation of exchange of sodium and alkalinity, thereby improving the physical shape of the soil. However, heavy metal elements in amendments often enter the soil along with beneficial components. These metal elements are not easy to migrate, remain for a long time, have strong concealment, and are highly toxic [5]. On the one hand, they may be absorbed by plants, affecting the normal growth and development of plants. development, and they may enter the food chain, threatening the health of humans and the reproduction of other animals. On the other hand, they may enter groundwater with surface runoff, posing a threat to environmental safety and causing irreversible pollution. Therefore, in the process of applying chemical modifiers, it is necessary to ensure an appropriate amount.

\section{Physical Improvement Measures}

The physical measures of saline-alkali land improvement mainly adjust the interaction between water and salt in the soil by changing the physical structure of the soil, and finally achieve the purpose of inhibiting soil evaporation and improving the effect of salt leaching and infiltration. It mainly includes improvement methods such as leveling the land, deep plowing, raising the terrain, irrigation and drainage system flushing and desalination, and sand pressing. Salt washing is to dissolve the salt in the soil through irrigation, and use the water infiltration process to discharge the soluble salt on the soil surface to the lower soil layer, and finally discharge the salt in combination with the drainage system [6]. Cutting the bottom and leveling the land can make the water irrigated to the surface and the surface water generated by rainfall infiltrate evenly, reducing the damage of salinity and alkali . In deep ploughing, the soil with more surface salt is turned to the lower layer, and the soil with less salt in the lower layer is turned to the surface again [7]. Deep plowing and drying can cut off soil capillaries, reduce soil water evaporation, improve soil activity and fertility, and enhance soil permeability, thereby effectively controlling soil salt return. With the research and development of materials science, the method of using zeolite and ground cover to improve saline-alkali land has appeared. Zeolite has a wide range of sources, low cost, non-toxic and harmless, and has strong adsorption capacity and ion exchange capacity [8]. Adding zeolite to soil can not only play a role in maintaining fertilizer and supplying fertilizer, but also reduce soil alkalization. and salinity, and buffers the $\mathrm{pH}$ of the soil. The advantage of the physical improvement method is that it can effectively reduce the salt content in the soil, with high speed and high efficiency.

The intelligent transmission and data management control system of the Internet of Things is gradually applied in the management and construction of saline-alkali land management and utilization. Use information technology to scientifically manage and control project cost control, construction, and maintenance of demonstration bases, connect the Internet of Things through testing equipment, use professional software and more advanced methods to process the collected data, and realize the information management of saline-alkali land sorting and utilization. Coverage is an important quantitative index to measure the structure of plant community. The traditional measure of grassland vegetation coverage adopts the grid method, and now the digital image method is being promoted. It has the advantages of small error, high accuracy and simple operation, and can be applied Based on quadratic measurements of grassland vegetation coverage. For large-area vegetation coverage measurement, remote sensing images or high-altitude digital aerial photography can be used to estimate grassland vegetation coverage more accurately and conveniently. Therefore, the digital image method is expected to become the main method of grassland vegetation coverage measurement in the future.

\section{Water Conservancy Improvement Technology}

The water conservancy improvement technology mainly adopts the drainage method, which discharges the salt in the soil from the farmland, reduces the soil salt content, and provides a good water and salt environment for the growth of crops. At present, the main forms of farmland drainage include underground pipes, shafts, and open ditch drainage, etc., and underground underground pipes are mostly used to drain salt. According to the principle of "salt comes with water, salt goes with water; salt comes with water, water disperses salt and stays", it makes the salt in the soil drain away with water, and controls the groundwater level below the critical depth to achieve soil desalination and The purpose of preventing secondary salinization is to improve the physical environment of the topsoil. Concealed pipe drainage has the advantage of occupying less cultivated land, reducing bridges and culverts and facilitating mechanized farming [9]. Han Jichang et al. summarized the practice and skillfully used the scientific mechanism of water-salt balance. By laying out the water storage ditch system, adjusting the water level change of the storage ditch to accelerate the convective and dispersing effect of salt, and developed a "storage" as the core. "The technology of water storage and salt pressure in saline-alkali land realizes the dynamic circulation between water and soil to press salt [10].

\section{Biological Improvement Measures}

Among the various technologies of saline-alkali land treatment, biological measures are generally considered to be the most effective improvement methods, mainly by planting salt-tolerant plants in saline-alkali land, leaching soil salt to the lower soil through irrigation, and controlling the salt below the plant root system Soil layer, improve the soil structure of the plough layer, increase soil fertility, and gradually improve soil physical and chemical properties. The development of salt-tolerant plants can not only improve soil productivity and reduce the cost of using high-quality irrigation water, but also 
contribute to a virtuous cycle of agroecology and environmental improvement in saline environments [11]. Since the 1930s, scholars in many countries have begun to pay attention to the study of salt tolerance of plants. Since the 1980s, my country has also carried out research and screening on the salt tolerance of plants, and has achieved certain results. Many scholars have successfully planted salt-tolerant crops such as Casuarina, Populus euphratica, Seabuckthorn, Zhumei Begonia, Sand jujube and other salt-tolerant tree species, as well as Suaeda salsa and Salvia chinensis in saline-alkali land. While planting salt-tolerant crops in saline-alkali land, screening of halophytes was also carried out [12], such as the cultivation of salt-tolerant rice, potato, wheat, sorghum, tomato and other varieties. There are also studies on the improvement of different saline-alkali wastelands using Xingxingcao and D. koreanica, and the benefits of soil improvement are also very significant. These studies have greatly contributed to biological measures for salinealkali land improvement. At the same time, combining planting and aquaculture to improve saline-alkali land with biological cycle as a mechanism has good economic benefits [13].

Microorganisms are the most active components in soil and play an important role in soil occurrence, development and development. Some studies have shown that the microbial fertilizer can change the physical and chemical properties and biological species of the soil, the application of microbial inoculants can promote the number and species of soil heavy microorganisms, and the organic acids generated in the metabolic activities of microorganisms can affect the alkalinity of saline-alkali soils. Neutralization, a variety of enzymes produced by the activities of microorganisms can promote the decomposition and synthesis of soil organic matter, and reduce the fixation of phosphorus and potassium in the soil, which is conducive to the transformation of insoluble phosphorus and potassium. Some studies have used microbial fertilizers to replace traditional chemical fertilizers, and carried out afforestation tests on soda saline-alkali land. The results show that the plant survival rate and preservation rate of microbial fertilizers have been improved, and microbial fertilizers have effectively improved saline-alkali land and showed long-term effectiveness[14].

\section{Conclusion}

Most of the technical measures for the improvement of saline-alkali land are to reduce the soluble salt content in the soil through desalination and salt removal. Methods such as water conservancy engineering measures, agricultural farming measures, halophytes, and chemical modifiers have been widely used. Although chemical improvement measures can quickly change soil structure, they may also bring about secondary pollution; agricultural measures require a lot of manpower, material and financial resources; biological improvement measures are slow to take effect and have a long cycle. Due to the different regional distribution of saline-alkali lands, different geological conditions, climate differences and other factors resulting in different saline-alkali geography characteristics, for the saline-alkali lands of different soil qualities and different regions, it is necessary to take the improvement measures of "adjusting measures to local conditions and comprehensive prevention and control". As an important land reserve resource, saline-alkali land has been paid more and more attention. The effective use of saline-alkali land will play an important role in ensuring food security, promoting rural revitalization, and promoting the construction of ecological civilization[15]. However, there are still some problems in the key technologies of saline-alkali land management that need to be solved. How to turn salinealkali land into a usable land resource is the main ecological and environmental problem facing agriculture in arid and semi-arid areas, and it is also an effective way to ease the tension of land resources.

\section{Acknowledgments}

Project Fund: DJNY2021-26 Internal Research Project of Shaanxi Land Engineering Construction Group.

\section{References}

1. Uzoma K C, "Inoue M,Andry H,et al.Effect of cow manure biochar on maize productivity under sandy soil condition".Soil Use and Management,2011,27(2):205-212.

2. Yang L Q.“Xinjiang Saline-Alkaline Soil Resources and Comprehensive Management".Chinese Journal of Soil Science,1993(s1):15-17.

3. Bai $\mathrm{X}, \mathrm{Wu} F \mathrm{~F}$, Lin F M, et al."Establishment of Soybean Salt Tolerance Identification System". Journal of Northeast Agricultural University,2013, 44(4):8-13.

4. Liu C H,Zhang W S'Study on Salt Tolerance of Sixty-nine Alfalfa Varieties and Two Physiological Indicators of Salt Tolerance".Pratacultural Science, 1993(6):1-6.

5. Lu J"Remediation mechanism and application study of improver in heavy metal contaminated soil"China Energy and Environmental Protection,2018, 40(6):103-107.

6. Wang S J, Chen Q, Li Y, et al.“Experimental Investigation on Distribution Profiles of Heavy Metals in the Desulfurization Gypsum Amended Saline and Sodic Soils".Ecology and Environmental Sciences, 2013, 22(5): 851-856.

7. Wang J C,Yin L"Technical measures for improving saline-alkali land"Modern Agricultural Science and Technology,2011(12):282-282 .

8. Ma C,Ma L Y,Liu T X,et al."Research progress on improvement and utilization technology of salinealkali land".World Forestry Research,2010,23(2):2832.

9. Wang H J,Li C,Zhong X M,et al."Research progress of saline-alkali land improvement 
technology".Agricultural

Engineering,2014,4(s1):44-47.

10. Han J C,Xie J C,Zhu J W,et al."Study on Comprehensive Management Model of Saline-Alkali Land in Lubotan, Shaanxi ".Journal of Hydraulic Engineering,2009,40(03):372-377.

11. Niu D L,Wang Q J.“Research progress of salinealkali land management ". Chinese Journal of Soil Science,2002,33(6):449-455.

12. Lv Z S,Wang R Y."Preliminary Study on Fuzzy Comprehensive Evaluation of Effects of Different Biological Measures on Soda Salt Soil Improvement".Chinese Journal of Soil Science, 1989,20(5):200-205.

13. Jin J Y,Zhang N,Liang M Z,et al.“Application of systematic research method of soil nutrient status in soil fertility research and soil testing and fertilization "Journal of Plant Nutrition and Fertilizers, 1996(1):8-15.

14. Tian X L."Micro-organisms fertilizer and its effectiveness evaluation improvement saline-alkali soil in Daqing"Northeast Forestry University,2010.

15. Zhang H O,Shi C D."Brief Analysis of Saline-Alkali Land Treatment Technology ".Rural Economy and Science-Technology,2021,32(13):7-9. 